\title{
Blood pressure lowering effects of a novel isometric exercise device following a 4-week isometric handgrip intervention
}

This article was published in the following Dove Press journal: Open Access Journal of Sports Medicine

\author{
Daniel S Baddeley-White' \\ Cheri L McGowan ${ }^{2}$ \\ Reuben Howden ${ }^{3}$ \\ Benjamin DH Gordon ${ }^{4}$ \\ Peter Kyberd ${ }^{5}$ \\ Ian L Swaine ${ }^{1,2}$ \\ 'Department of Life \& Sport Sciences, \\ University of Greenwich, Medway \\ Campus, London, UK; ${ }^{2}$ Department of \\ Kinesiology, Faculty of Human Kinetics, \\ University of Windsor, Windsor, ON, \\ Canada; ${ }^{3}$ Department of Kinesiology, \\ University of North Carolina at \\ Charlotte, Charlotte, NC, USA; \\ ${ }^{4}$ Department of Exercise and \\ Rehabilitative Sciences, Slippery Rock \\ University, Slippery Rock, PA, USA; \\ ${ }^{5}$ Department of Engineering Science, \\ University of Greenwich, Medway \\ Campus, London, UK
}

Background: Hypertension is the leading risk factor for global mortality. Isometric resistance exercise training reduces blood pressure (BP). However, the protocols used are often limited by cost/immobility and the use of rigid exercise modalities. In response, a novel more versatile, isometric exercise (IE) device, the IsoBall (IB) was created.

Purpose: The aim of this study was to test the BP-lowering effectiveness of this prototype. Methods: Twenty-three healthy participants $(29.10 \pm 2.19$ years old, $173.95 \pm 3.83 \mathrm{~cm}, 75.43$ $\pm 5.06 \mathrm{~kg}$, SBP $127.10 \pm 10.37 \mathrm{mmHg}$, DBP $70.40 \pm 6.77 \mathrm{mmHg}$ ) were randomly allocated to either a control group (CON) or 2 isometric handgrip (IHG) training groups that used the Zona plus (ZON) and IB devices. The intervention groups completed 3 sessions each week of 4, 2 min IHG at $30 \%$ maximal voluntary contraction, with a 1-min rest, for 4 weeks. Resting BP, heart rate (HR) and IHG strength were measured in all groups at baseline and postintervention.

Results: Postintervention systolic BP (SBP) was significantly lower in both ZON (114.5 \pm 8.2 $\mathrm{mmHg}, p=0.000)$ and IB $(119.9 \pm 7.0 \mathrm{mmHg}, p=0.000)$ compared to control $(131.0 \pm 12.4$ $\mathrm{mmHg}$ ). Postintervention diastolic BP (DBP) was reduced in both intervention groups (ZON $66.6 \pm 7.4 \mathrm{mmHg}, p=0.004$; IB $65.7 \pm 10.0 \mathrm{mmHg}, p=0.012)$ compared to $\mathrm{CON}(71.1 \pm 8.8$ $\mathrm{mmHg}$ ). Mean arterial pressure (MAP) was reduced in both groups (ZON $82.6 \pm 6.8 \mathrm{mmHg}$, $p=0.000 ; \mathrm{IB} 84.3 \pm 9.1 \mathrm{mmHg}, p=0.000)$ compared to control $(91.0 \pm 9.7 \mathrm{mmHg})$. No significant changes were seen in HR or strength $(p>0.05)$.

Conclusion: The results of this study indicate that both the ZON and IB devices elicit significant SBP, DBP and MAP reductions. Despite the ZON group having larger reductions in BP, no significant differences were found between the two devices. Thus, this study indicates the IB device to be an effective alternative to the $\mathrm{ZON}$ that can also be used to perform other IE modalities.

Keywords: hypertension, antihypertensive intervention, isometric exercise ball, IsoBall, Zona plus device

\section{Introduction}

Globally, hypertension is estimated to affect $\sim 30 \%$ population $^{1}$ and is the leading risk factor for global mortality causing an estimated 9.4 million deaths a year. ${ }^{2}$ Hypertension is a major risk factor in the development of cardiovascular disease (CVD) and coronary heart disease (CHD). ${ }^{3,4}$ A reduction in blood pressure (BP) is associated with a reduced risk of developing CVD. ${ }^{5,6}$ The significant public health burden that hypertension represents highlights the importance of effective antihypertensive treatments.

Despite the proven efficacy of pharmacological antihypertensive treatments, ${ }^{7}$ less than $50 \%$ of medicated hypertensives adhere to treatment for numerous
Correspondence: Daniel S BaddeleyWhite

Department of Life \& Sport Sciences, University of Greenwich, Medway Campus, Central Ave, Gillingham, Kent ME4 4TB, UK

Email db|9291@gre.ac.uk 
reasons, including deleterious side effects, and many often fail to achieve clinical targets. ${ }^{8-10}$ These, together with the high cost of hypertension to the national health service (NHS), make lifestyle modification treatments essential. Lifestyle modification treatments are a widely recommended approach to reduce BP. ${ }^{11}$ These interventions include weight reduction, dietary alterations including sodium and alcohol restriction, and increased physical activity. ${ }^{12}$ These treatments have no deleterious side effects and are comparatively low in cost. ${ }^{13}$

As noted above, increased physical activity is a cornerstone lifestyle modification for hypertension management, with guidelines around the world overwhelmingly recommending aerobic exercise training with dynamic resistance training as an adjuvant intervention. ${ }^{14-18}$ Recently, isometric handgrip (IHG) training (a form of isometric resistance exercise training (IET)) has been introduced into formal guidelines, most notably by the American College of Cardiology and the American Heart Association in their 2017 Guidelines. ${ }^{14}$ To date, IET interventions have consisted of a limited number of exercises, with the most studied being IHG interventions. ${ }^{19-27}$ IET generally consists of four, 2-minutes (min) isometric contractions, separated by brief rest periods. ${ }^{28}$ IHG training is most often performed at $30 \%$ of maximal voluntary contraction (MVC), three times per week, ${ }^{14,28}$ over a period of $8-10$ weeks $;{ }^{28}$ however, several studies have reported significant BP reductions using shorter, 4-week, intervention. ${ }^{23,24,27,29,30-32}$ Other protocols, such as double and single leg extensions, ${ }^{27,29,33-35}$ isometric wall squat $^{30}$ and the maximal intermittent (MINT) handgrip protocol $^{36}$ have also been found to reduce BP.

Numerous randomized control trials and meta-analyses have found IET to elicit significant reductions in resting blood pressure (RBP) in those with and without hypertension. ${ }^{19-21,27,29,30,37,38}$ Early evidence suggests that IET also reduces 24-hours (hrs) ambulatory BP. ${ }^{39}$

As with many BP-lowering treatments, and for reasons not yet entirely understood, not everyone responds to IET similarly. Inherent participant characteristics together with training intensity may all play a role in response efficacy. For example, individuals with higher pre-training RBP, older individuals and individuals who have a high pre-training BP reactivity response appear most responsive to IET. ${ }^{19,27,40-42}$ IET appears ineffective in well-controlled medicated hypertensives ${ }^{25}$ and the role of gender is unclear $;^{19,39,41}$ however, there is evidence to suggest that older women may be more responsive. ${ }^{43}$ With respect to the latter, the equivocal nature of the findings to date may reflect the higher initial gender-specific baseline pressures and/or the small number of women collectively studied to date in IET trials. Finally, there is evidence to suggest that low-intensity short duration IET (isometric leg) interventions may be insufficient to cause RBP reduction. ${ }^{33,44}$

Regarding IET's efficacy compared to other exercise interventions, Cornelissen et $\mathrm{a}^{45}$ noted reductions of 13.5 $\mathrm{mmHg}$ systolic pressure and $6.1 \mathrm{mmHg}$ diastolic pressure following IET, with post-training reductions of $\sim 5 / 4$ mmHg cited in the most recent meta-analysis. ${ }^{19}$ These reductions are similar to those seen in traditional dynamic exercise interventions, both aerobic and resistance, despite the lower time to perform the exercise bouts. ${ }^{37}$ The lower time commitment (as little as $12 \mathrm{~min}$ ) coupled with the simplicity of the intervention, and the lower cost, may contribute to increased adoption of and adherence to the intervention. ${ }^{37}$ Making IET interventions, particularly IHG training, more applicable for those individuals with mobility issues. It should also be noted that IET interventions have been found to yield beneficial results when performed unsupervised in the participants home, ${ }^{30}$ which may further contribute to increased adherence rates.

Each IET program utilizes equipment and protocols designed specifically for that exercise (eg, handgrip dynamometers), or immobile equipment (isokinetic dynamometers) that are expensive and/or not available outside medical/academic institutions. These factors may restrict accessibility to IET and those with mobility limitations may not be able to perform specific IET exercises or travel to a location with specialized equipment. In response, a multidisciplinary research team at the University of Greenwich has developed a versatile isometric device that can be used to perform any number of isometric exercises (IE). This work has resulted in the development of a prototype novel IE device designated the IsoBall (IB). The IB device was developed using several criteria, including low cost, high versatility and ease of transport/mobility. These criteria were used in order to increase the applicability, accessibility, adherence and initiation rates. ${ }^{46-48}$

The aim of the current study was to investigate the concept and efficacy of this device using the most widely studied and efficacious IET protocol (IHG training, four, 2-min contractions separated by a 1-min rest at $30 \% \mathrm{MVC}$, 
3 times a week ${ }^{14,28}$ ), and the one included in the ACC/ AHA 2017 Guidelines. ${ }^{14}$ It was hypothesized that the IB device would elicit similar significant BP reductions when compared to a traditional computerized handgrip dynamometer following a 4-week intervention.

\section{Method}

Twenty-three healthy participants were recruited via poster and email advertisements (Table 1). Sample size was calculated using $G^{*}$ power (version 3.1.9.2, Germany). Effect size data, relating to SBP, form several meta-analysis was used to estimate sample size. ${ }^{19,37,45}$ The results of this analysis indicate the need for $n=26$, to achieve a $\beta \geq 0.95$ and $\alpha \leq 0.05$. Participants reported not taking any medications known to effect cardiovascular function during the study. All female participants reported using oral contraception. Ethical approval was granted by the University Research Ethics Committee (UREC/16.2.5.14) at the University of Greenwich

After providing written informed consent, participants were randomly allocated to one of three groups, using a counterbalanced simple randomization method by a thirdparty researcher. These groups were the control $(\mathrm{CON})$ group, the ZON group that completed the intervention using the Zona plus (Zona plus Series 3, Zona Health Inc,

Table I Participants' baseline characteristics

\begin{tabular}{|c|c|c|c|c|c|c|}
\hline & \multicolumn{2}{|l|}{ CON } & \multicolumn{2}{|l|}{ IB } & \multicolumn{2}{|l|}{ ZON } \\
\hline & Male $(\mathrm{N}=5)$ & Female $(\mathbf{N}=3)$ & Male $(\mathrm{N}=4)$ & Female $(\mathbf{N}=3)$ & Male $(\mathrm{N}=4)$ & Female $(\mathrm{N}=4)$ \\
\hline Stature $(\mathrm{cm})$ & $174.8 \pm 10.5$ & $170.1 \pm 5.2$ & $178.0 \pm 6.2$ & $177.0 \pm 7.2$ & $175.5 \pm 6.9$ & $168.5 \pm 13.4$ \\
\hline Mass (kg) & $74.0 \pm 12.7$ & $68.9 \pm 17.1$ & $82.7 \pm 8.2$ & $79.5 \pm 6.1$ & $75.8 \pm 7.2$ & $71.7 \pm 15.0$ \\
\hline Age (years) & $26.0 \pm 5.1$ & $27.3 \pm 3.1$ & $28.8 \pm 5.9$ & $29.7 \pm 0.6$ & $31.5 \pm 0.6$ & $31.3 \pm 6.0$ \\
\hline $\mathrm{RSBP}(\mathrm{mmHg})$ & $132.0 \pm 8.4$ & $123.7 \pm 24.5$ & $137.8 \pm 6.4$ & $118.0 \pm 1.7$ & $137.8 \pm 3.6$ & $113.3 \pm 3.5$ \\
\hline $\mathrm{RDBP}(\mathrm{mmHg})$ & $73.2 \pm 6.5$ & $68.3 \pm 14$ & $77.3 \pm 6.0$ & $60.3 \pm 8.0$ & $77.3 \pm 3.3$ & $66.0 \pm 9.6$ \\
\hline RHR (bpm) & $71.8 \pm 8.8$ & $73.7 \pm 10.2$ & $65.0 \pm 4.3$ & $65.7 \pm 5.7$ & $66.0 \pm 7.5$ & $57.3 \pm 12.7$ \\
\hline StrR (kg) & $29.6 \pm 10.3$ & $35.3 \pm 9.4$ & $34.2 \pm 14.0$ & $28.1 \pm 6.3$ & $38.1 \pm 10.7$ & $25.0 \pm 9.3$ \\
\hline StrL (kg) & $30.4 \pm 9.5$ & $37.1 \pm 11.1$ & $31.7 \pm 11.6$ & $25.3 \pm 7.1$ & $34.7 \pm 9.9$ & $27.7 \pm 10$ \\
\hline
\end{tabular}

Note: Data displayed as mean \pm standard deviation.

Abbreviations: CON, control; IB, IsoBall; ZON, Zona; RSBP, resting systolic blood pressure; RDBP, resting diastolic blood pressure; RHR, resting heart rate; StrR, righthandgrip strength; StrL, left-handgrip strength.

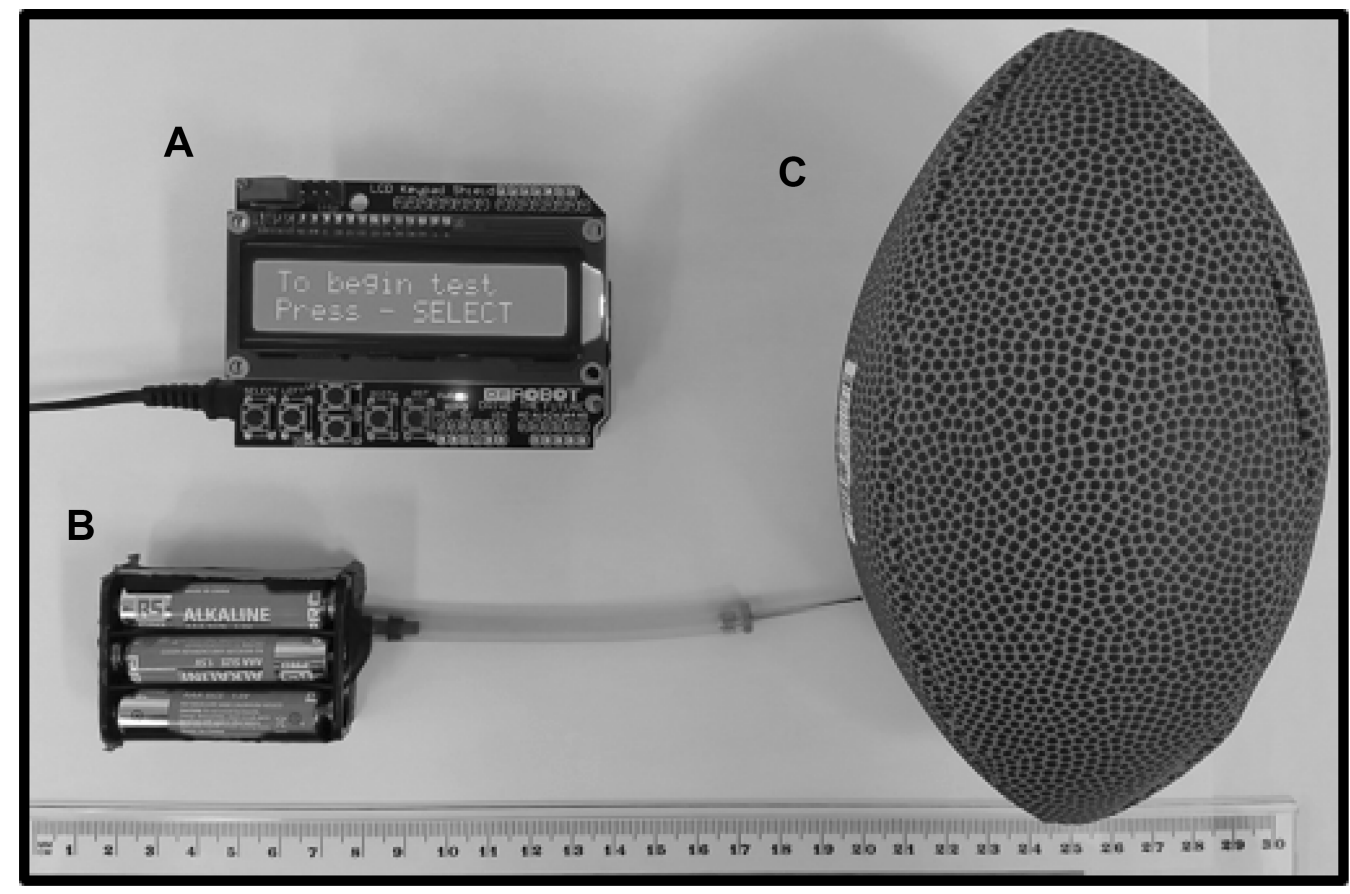

Figure I (A) IB visual display and processing unit. (B) IB pressure sensor unit with needle valve adaptor. (C) Mini size rugby ball. 
USA) device and the IB group that used the novel ball device (Figure 1) attached to a mini size Rugby ball (British \& Irish Lions replica, Rhino, UK) to complete the intervention. Intervention groups' familiarization sessions consisted of RBP measurements, handgrip strength tests (Str) and 1 bout of IHG exercise (four, 2-min IHG contractions performed at $30 \%$ MVC using alternating hands, with each IHG contraction separated by a 1-min rest period). Participants completed the familiarization sessions using either the IB or the ZON depending upon group allocation. Control group familiarization sessions consisted of only RBP measures, as the use of IET has been found to effect BP after one session. ${ }^{49}$ Two familiarization sessions were used to ensure participants were completely comfortable with all protocols and with having their BP measured, these sessions were separated by $\geq 24$ hrs. Participant's stature (Seca 213 stadiometer, Seca GmBH, Germany) and mass (Seca 861 weight scales, Seca $\mathrm{GmBH}$, Germany) were measured during the first familiarization session. At least $24 \mathrm{hrs}$ after the last familiarization session, baseline testing occurred to assess RBP, heart rate (HR) and Str. After baseline testing, participants in the intervention groups (ZON; IB) were required to train 3 times per week for 4 weeks. All training sessions were completed within the University of Greenwich's laboratory, under supervision. Each training session involved four, 2-min IHG contractions at $30 \%$ MVC separated by $1 \mathrm{~min}$, each 2-min IHG contraction was performed in alternate hands. Post-training assessments of RBP, HR and Str were performed $\geq 24 \mathrm{hrs}$ following the last training session. Participants randomized to the CON group performed no IHG training and returned to the laboratory for post-testing 4 weeks following their pre-testing session. Each of the training and assessment sessions was separated by $\geq 24 \mathrm{hrs,}$ and sessions were conducted at the same time of day to avoid circadian rhythm effects.

Participants were instructed to abstain from caffeine and nicotine for $>2 \mathrm{hrs}$ prior to testing and from alcohol $>24 \mathrm{hrs}$ prior to testing. Participants were asked to maintain their habitual diet and exercise habits throughout the study period, and this was confirmed verbally prior to the initiation of each testing session. Participants were tested at the same of time of day, with the temperature controlled between $19^{\circ} \mathrm{C}$ and $23^{\circ} \mathrm{C}$, and ambient noise kept to a minimum.

\section{Equipment}

Briefly, the IB device works by sensing the pressure exerted on the ball attached to the pressure sensor via the needle valve adaptor (Figure 1B). This data is sent via Bluetooth to the visual display and processing unit (Figure 1A), which displays visual feedback to participants, informing them to either apply more or less pressure. Upon initial start-up of the device, an automatic MVC protocol is completed, consisting of a single 5-second (s) maximal contraction. The MVC data is used by the device to calculate the intensity (30\% MVC). Preliminary testing found the device to be highly correlated with calculated values $(\mathrm{y}=0.3277 \mathrm{x}$ $+1.0199 \mathrm{R}^{2}=0.9814$ ), indicating it to be an accurate method of controlling IE intensity.

\section{Measurement procedures}

\section{Resting blood pressure and heart rate}

RBP (systolic BP (SBP); diastolic BP (DBP)) and HR measurements were recorded from the participant's nondominant arm (Intellisense M3, Omron Healthcare Co, Japan), in a supine position. BP was collected supine, as this allowed for more comfortable and controllable positioning of the participants. The positioning was selected based on previous research. ${ }^{50,51}$ Briefly, Vischer and Burkard ${ }^{50}$ and Pickering et $\mathrm{al}^{51}$ describe how BP changes based upon body position, and note that whichever position (seated or supine) selected should be adhered to throughout all subsequent readings. Thus, the methods used for BP measurements were based upon these studies ${ }^{50,51}$ and collected as follows. Participants' supine with the BP cuff placed directly onto the skin so that the bottom of the cuff was $2-3 \mathrm{~cm}$ above the antecubital fossa area of the nondominant arm. The arm was supported so that it rested at the midpoint between the bed and the sternum to align with the right atrium. The participant was relaxed throughout with their legs uncrossed. All data was acquired following bladder voiding and a 10-min quiet rest period. Readings were taken in triplicate with a 60$\mathrm{s}$ period between each reading and the average of the three readings were calculated for data analysis. Mean arterial pressure (MAP) was calculated using the following equation:

$$
\mathrm{MAP}=\mathrm{DBP}+\left[\frac{1}{3}(\mathrm{SBP}-\mathrm{DBP})\right]
$$

\section{Handgrip strength}

IHG strength was measured using a digital hand grip dynamometer (Grip-D, T.K.K 5401, Takei, Japan) as per published protocol. ${ }^{52}$ Instantaneous MVC for each hand (right-handgrip strength (StrR) and left-handgrip strength (StrL)) were obtained in triplicate, alternating hand after each test. A 60-s rest period separated each reading. 


\section{Statistical analysis}

All statistical analyses were performed using Excel (Excel 2013, Microsoft office professional plus 2013, Microsoft corp, USA) and the Statistical Package for the Social Sciences (SPSS version 24, IBM corp, USA), with $\alpha$ set at $p \leq 0.05$. Shapiro-Wilk analysis was performed to establish data distribution. An ANCOVA test was performed to ascertain if there was a significant difference in the amount each group changed over the course of the intervention when controlling for baseline measures. Posthoc Bonferroni tests were performed to establish between group differences. Individual response data is reported using the methods described in Hopkins ${ }^{53}$; these data are reported in standardized units to $95 \%$ CIs.

\section{Results}

All participants in the intervention groups completed 12 IET sessions with a $100 \%$ adherence rate. No changes in diet or exercise were indicated. All outcome data were found to be normally distributed ( $p \geq 0.05$ ).

An ANCOVA test revealed that baseline SBP levels were significantly related to postintervention SBP levels $(F(1,23)=$ $79.63, p=0.000, r=0.95)$. After controlling for the covariate (baseline SBP) a significant effect was found $(F(2,23)=$ 24.14, $\left.p=0.000, \eta_{p}{ }^{2}=0.71\right)$. The post-hoc Bonferroni test indicates the significant difference to be between the intervention groups (IB, $119.9 \pm 7.0 \mathrm{mmHg}, p=0.000 ; \mathrm{ZON}, 114.5 \pm 8.2$ $\mathrm{mmHg}, p=0.000)$ and the control group $(131.0 \pm 12.4 \mathrm{mmHg})$. No significant difference was apparent between the IB and ZON groups ( $p=0.620$, see Figure 2$)$. Individual standardized SBP response data indicate the IB $(0.62 \pm 0.66)$ to have experienced a trivial to very large change and the ZON $(0.72 \pm 0.28)$ group experienced moderate to very large change.
Postintervention, DBP data was also found to be significantly related to baseline measures $(F(1,23)=229.259, p=$ $0.000, r=0.70)$. After controlling for baseline DBP levels, a significant effect was found $\left(F(2,23)=8.624, p=0.000, \eta_{\mathrm{p}}{ }^{2}\right.$ $=0.47)$. The post-hoc test revealed a significant difference $(p=$ $0.012)$ between postintervention IB $(65.7 \pm 10.0 \mathrm{mmHg})$ and CON $(71.1 \pm 8.8 \mathrm{mmHg})$; there was also a significant difference between CON and ZON (66.6 $7.4 \mathrm{mmHg}, p=0.004)$. No significant difference was found between the two intervention groups (IB and ZON, $p=1.000$, see Figure 3). Individual DBP response was trivial to large in the IB group $(0.46 \pm 0.43)$ and small to large responses in the ZON group (0.56 \pm 0.34$)$.

Postintervention MAP data was also found to be significantly related to preintervention MAP results $(F(1,23)=227.662, p=0.000, r=0.85)$. After controlling for this, a significant intervention effect was found $\left(F(2,23)=22.374, p=0.000, \eta_{p}{ }^{2}=0.70\right)$. The post-hoc tests revealed a significant difference between each intervention group (IB $84.3 \pm 9.1 \mathrm{mmHg}$, $p=0.000 ; \mathrm{ZON} 82.6 \pm 6.8 \mathrm{mmHg}, p=0.000)$ and control $(91.0 \pm 9.7 \mathrm{mmHg})$. No significant difference was found between the intervention groups $(p=0.750)$. Figure 4 . MAP individual responses indicate the IB to cause trivial to large $(0.50 \pm 0.43)$ responses, with small to very large responses seen in the ZON group $(0.63 \pm 0.54)$.

No significant differences $(p>0.05)$ were observed in any other parameters measured. Data values for all parameters collected over the 4-weeks of IET intervention are summarized in Table 2.

\section{Discussion}

The primary aim of this study was to evaluate the concept and efficacy of the prototype IB using the widely studied

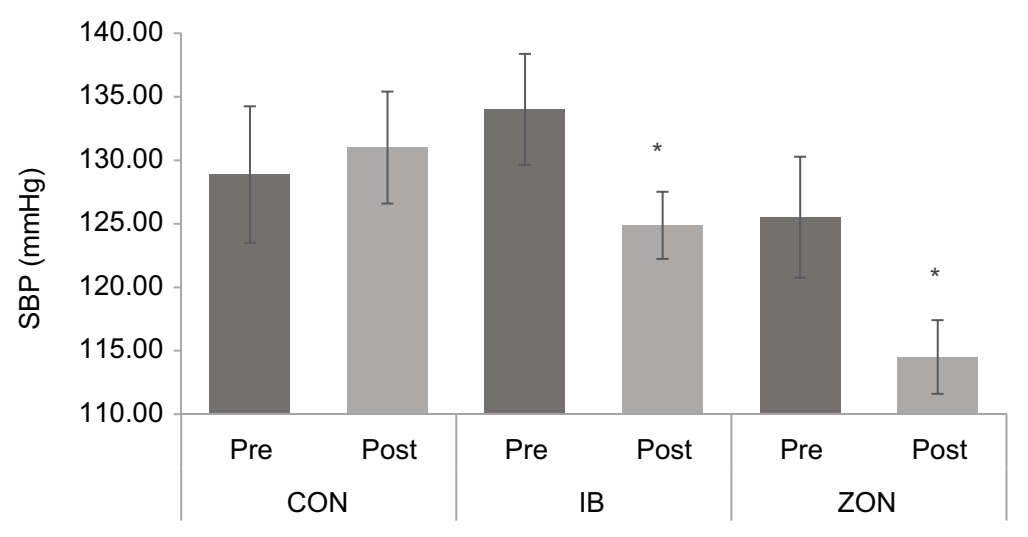

Figure 2 Pre- to post-systolic blood pressure (SBP) mean \pm standard error.

Note: *Indicates significance $(p<0.05)$.

Abbreviations: CON, control; IB, IsoBall; ZON, Zona. 


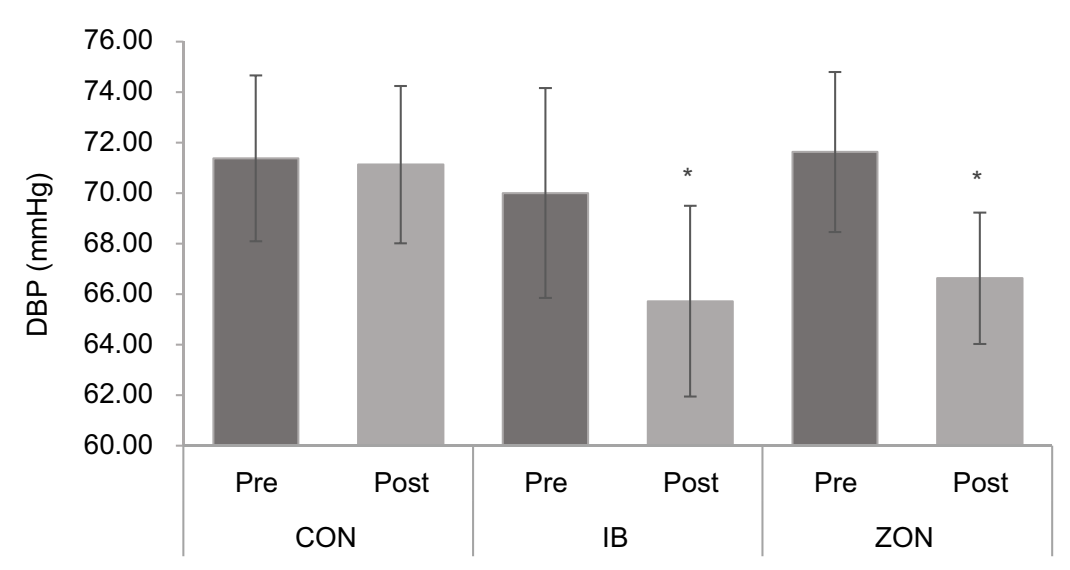

Figure 3 Pre- to post-diastolic blood pressure (DBP) mean \pm standard error.

Note: *Indicates significance $(p<0.05)$.

Abbreviations: CON, control; IB, IsoBall; ZON, Zona.

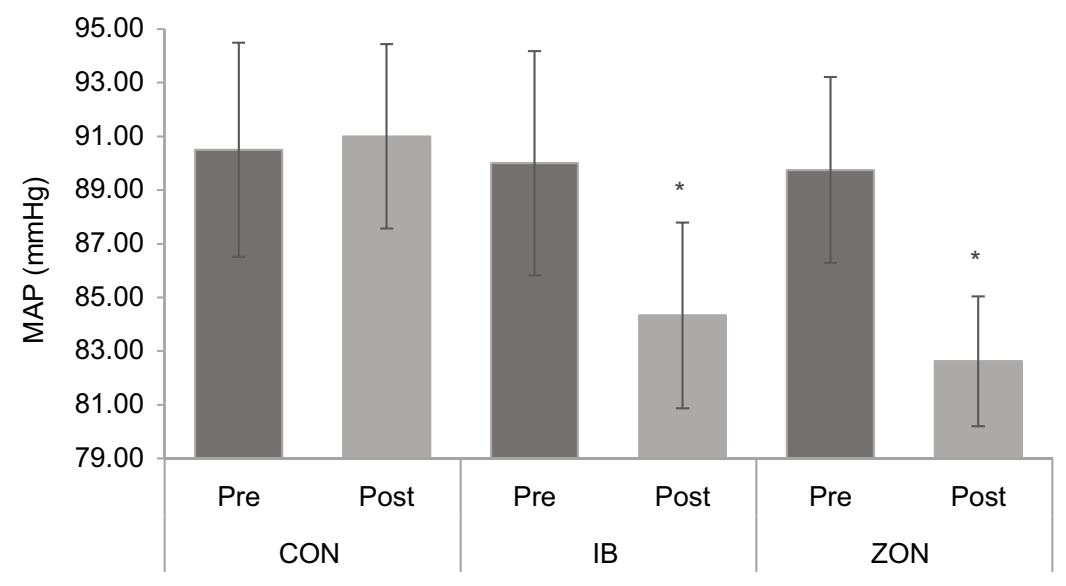

Figure 4 Pre- to post-mean artierial pressure (MAP) mean \pm standard error.

Note: *Indicates significance $(p<0.05)$.

Abbreviations: CON, control; IB, IsoBall; ZON, Zona.

Table 2 Mean and SD values for all parameters measured, for each group, preintervention and postintervention

\begin{tabular}{|l|l|l|l|l|l|l|}
\hline \multirow{2}{*}{ Parameters } & \multicolumn{2}{l|}{ CON } & IB & \multicolumn{2}{l|}{ ZON } \\
\cline { 2 - 7 } & Pre & Post & Pre & Post & Pre & Post \\
\hline SBP (mmHg) & $128.9 \pm 15.2$ & $131.0 \pm 12.4$ & $129.3 \pm 11.5$ & $119.9 \pm 7.0^{*}$ & $125.5 \pm 13.5$ & $114.5 \pm 8.2^{*}$ \\
DBP (mmHg) & $71.4 \pm 9.3$ & $71.1 \pm 8.8$ & $70.0 \pm 11.0$ & $65.7 \pm 10.0^{*}$ & $71.6 \pm 9.0$ & $66.6 \pm 7.4^{*}$ \\
MAP (mmHg) & $90.5 \pm 11.3$ & $91.0 \pm 9.7$ & $90.0 \pm 11.0$ & $84.3 \pm 9.1^{*}$ & $89.8 \pm 9.8$ & $82.6 \pm 6.8^{*}$ \\
HR (bpm) & $72.5 \pm 8.7$ & $74.6 \pm 8.7$ & $65.3 \pm 4.5$ & $66.4 \pm 4.0$ & $61.6 \pm 10.7$ & $60.9 \pm 10.6$ \\
StrR (Kg) & $31.7 \pm 9.7$ & $32.8 \pm 9.9$ & $31.6 \pm 11$ & $33.5 \pm 12.3$ & $31.6 \pm 11.6$ & $34.0 \pm 11.8$ \\
StrL (Kg) & $32.9 \pm 9.9$ & $31.8 \pm 10.3$ & $29.0 \pm 9.8$ & $32.2 \pm 11.5$ & $31.2 \pm 10.0$ & $32.6 \pm 11.6$ \\
\hline
\end{tabular}

Note: *Significant $(p<0.05)$ difference in pre- to post-change values compared to control.

Abbreviations: CON, control; IB, IsoBall; ZON, Zona; SBP, systolic blood pressure; DBP, diastolic blood pressure; MAP, mean arterial pressure; HR, heart rate; StrR, righthandgrip strength; StrL, left-handgrip strength.

IHG training protocol against a commercially available equivalent (ZON). Despite the ZON eliciting larger BP reductions in all parameters, the non-significant differences between the two devices confirm the hypothesis that both devices would elicit similar significant BP reductions compared to control. The BP results presented in this study are in line with previous IHG studies conducted over similar durations of 4-weeks, ${ }^{24,27} 5$-weeks, ${ }^{24,54}$ and 6-weeks. ${ }^{21,55,56}$ It is 
difficult to say whether the increase in control group SBP values contributed to the statistical significance of the reductions after isometric training. The reductions in both of the isomeric training groups were of a magnitude that was similar (if not greater) than many of the previous intervention studies, which average approximately $5.99 \mathrm{mmHg} \mathrm{SBP}$ and $3.94 \mathrm{mmHg}$ DBP reduction. ${ }^{19,37}$

It should be noted that BP reductions following IHG appear not to be associated with age or gender in young, healthy adults. ${ }^{40}$ This can be seen in studies that have directly compared male and female participants and deduced no gender difference. Somani et $\mathrm{al}^{39}$ and Hanik et $\mathrm{al}^{57}$ noted no difference in the magnitude of BP reduction following IHG between male and female participants, indicating IET to be equally effective in both genders. However, these studies were performed using young (mid-twenties) participants. In older postmenopausal women, greater BP reductions have been found than those seen in age-matched men. ${ }^{41,43,58}$ These findings are in agreement with previous research showing that the amount to which BP is reduced by IET is dictated by initial BP. ${ }^{19,40}$ That is to say, participants with higher initial BP experience greater reductions post-IET. Thus, it could be concluded that hypertensives will experience greater reductions in BP than normotensives. Due to the small sample size and baseline differences apparent between the male and female participants within this study, no investigations or conclusions can be conducted or drawn from the current data.

Regarding HR and handgrip strength, the results of the present study are consistent with the majority of previous IHG research in normotensive and hypertensive populations in finding no significant change in HR or strength. ${ }^{22-27,41,59}$ These results may be due to the intensity used not causing a great enough stimulus to elicit strength gains. Indeed, the current study and those that have previously reported no change in handgrip strength have all utilized similar IHG protocols. ${ }^{22-27,41,59}$ This is however, a speculative comment and studies investigating this phenomenon are warranted.

As well as statistical significance, it is also important to consider clinical importance, in order to convey further clinical insight. ${ }^{60,61}$ Clinical importance is established using Minimal Clinically Important Difference (MCID). ${ }^{60}$ MCID is considered the smallest effect required to produce clinically important results. ${ }^{62}$ Limited data is available on MCID in regard to BP; however, several papers have found reductions of $\geq 2 \mathrm{mmHg} \mathrm{SBP}$ and DBP could reduce $\mathrm{CHD}$ and stroke risk. ${ }^{63,64}$ Neaton et al. 1995 cited in ${ }^{6565}$ Indeed, Cook et al ${ }^{64}$ noted a $2 \mathrm{mmHg}$ DBP reduction would result in a $17 \%$ reduced risk of hypertension, $6 \%$ reduction in
CHD risk and a $15 \%$ reduction in stroke risk. Neaton et al (1995 cited in ${ }^{65}$ ) estimated this same reduction $(-2 \mathrm{mmHg}$ SBP) would reduce cardiovascular disease risk by $5 \%$ and all-cause mortality by $3 \%$. These potential effects were also estimated in Stamler et al ${ }^{65}$ noting a $2 \mathrm{mmHg}$ SBP reduction leading to $-4 \%$ risk of $\mathrm{CHD},-6 \%$ risk of stroke and $-3 \%$ risk of all-cause mortality. Using $\geq 2 \mathrm{mmHg}$ as the MCID for both SBP and DBP, the results of the present study show that $93.33 \%$ of the participants in the intervention groups achieved the $\geq 2 \mathrm{mmHg}$ SBP goal $(87.50 \% \mathrm{ZON}$ and $100 \%$ IB). A $86.66 \%$ experienced a $\geq 2 \mathrm{mmHg}$ DBP in both intervention groups (87.50\% ZON and 85.71\% IB). These findings are in line with previous literature that noted MCID rates of $60-96 \%$ in unmedicated individuals. ${ }^{37}$ Despite these MCID data, individual response data indicate there to be a small to very large effect on SBP when IHG is performed with either the IB or the ZON devices. Small to very large effect on ZON DBP and MAP data, with trivial to large DBP and MAP changes, is seen in the IB group data. These large variations in the individual response data are likely due to the small sample size.

One aspect of the IB that differentiates it from other IE devices is its versatility. The IB device can be attached to any ball via a needle valve adaptor; this enables the shape and size of the ball to be changed allowing for a wide variety of possible exercises to be performed. Such versatility may have adherence implications. Previous exercise adherence research has noted several factors that may dissuade individuals from initiating and maintaining physical activity programs. Factors include lack of time, boredom, location and difficulty of the exercise. ${ }^{46,47,48}$ All of these factors are overcome with IE as the interventions used require a short time commitment, at a low intensity and can be performed virtually anywhere. The final aspect, variety of exercise, is yet to be tested. Despite this, numerous IE modalities have been used to elicit BP reductions. Most common among these exercises is $\mathrm{IHG}^{20-27,39,41,66}$; however, both single leg and double leg extension have also been used to significantly reduce $\mathrm{BP},{ }^{33-35,44,67,68}$ with other, more novel, exercises also appearing in the literature, for instance, Howden et $\mathrm{al}^{68}$ found isometric arm curls efficacious at reducing BP, Bentley et al36,69 noting significant BP reductions with a high-intensity handgrip and MINT protocols and Wiles et $\mathrm{al}^{30}$ noting significant reductions following an isometric wall squat intervention. This variety of IE used in the previous research alludes to the plausibility that a multiexercise isometric program may be efficacious at reducing BP as well as having a positive influence on adherence rates. This remains to be tested; however, 
the IB device offers a method of controlling isometric intensity throughout a variety of exercise protocols, thus offering a lowcost and mobile method of conducting/prescribing such an intervention.

As the IB device was developed as an alternative to the currently available isometric intensity controlling devices, it is worth briefly comparing the IB to other devices. The IB prototype was developed at a build cost of approximately $£ 150$ which is substantially less than the Zona plus series 3 device (used in this study) that is priced at $£ 549.00$. $^{70}$ Currently, the Zona device is similar in size to the IB and thus is as mobile. The Zona is, however, limited as only one type of IE (IHG) can be performed with the device, thus limiting its use by individuals with hand mobility issues such as arthritis. Low-cost grip dynamometers can be purchased for $\sim £ 20$ and IHG can be performed with these devices; yet, they are limited to one type of IE and can be cumbersome. More versatile devices such as isokinetic dynamometers are viable options for performing a variety of IE; however, these devices are also limited by both cost and immobility limiting their applicability. Other more novel devices such as the bend and squat device ${ }^{30}$ are interesting options; however, again, this device is limited to one specific type of IE.

The next stage of the development of the IB is to link it to a smartphone application via a Bluetooth link. This has the potential to decrease the cost of a production version, allowing greater flexibility in the display, tracking of performance and increasing patient compliance with engagement through games.

The main limitation of the study was our slightly smaller than target sample size. Despite failing to recruit the estimated sample size by three participants ( $\mathrm{n}=23$ vs 26 ), the study proceeded as a proof of concept/pilot study. Importantly, a post-hoc power analysis using SBP as the primary measure indicated a power of 0.99 with the achieved sample size of $\mathrm{n}=23$. Another limiting factor of the study is the lack of menstrual cycle control. Generally, BP fluctuates with the phases of the menstrual cycle, with higher BP occurring during the follicular phase (0- to 14-day postmenstruation) and lower BP during the luteal phase ( -14 to -1 days prior to menstruation) ${ }^{71}$ In normotensive participants, fluctuations of $\pm 5.2 \mathrm{mmHg}$ SBP and $\pm 2.1 \mathrm{mmHg}$ DBP have been found, ${ }^{72}$ with other studies finding smaller ranges of $\pm 1.45 \mathrm{mmHg}$ SBP and $\pm 0.55 \mathrm{mmHg}$ DBP. ${ }^{71}$ It is likely that this factor influenced the results of this study; however, as the participant population consisted of both men and women, research has shown that this effect is smaller than the BP reduction found in this study. Given that numerous other IHG studies, conducted using both male and female participants, have found similar effects to those presented in this study, ${ }^{20,22,23,27,57}$ it is unlikely that the results presented here are entirely due to this factor. Finally, the inclusion of a placebo/sham intervention group would have strengthened the results of this study. However, due to the use of a proven device (ZON), it was concluded that this would be unnecessary. It should be noted that this is a common issue to IE research with very few studies including a sham/placebo group.

Further studies should be conducted using the IB device, and these studies should aim to increase the evidence for the efficacy of the IB device and utilize the adaptability of the device to create a variety of effective IE programs. Studies should also aim to investigate the efficacy of a multiexercise isometric program. Further studies should also continue to investigate the efficacy of home-based interventions as these data remain scarce.

\section{Conclusion}

In conclusion, despite the $\mathrm{ZON}$ eliciting greater reductions in BP, the novel IE device (IB) also elicited significant reductions in all resting BP parameters similarly to the ZON with no significant differences between the two devices. Therefore, the IB device should be further tested and used in future studies as a diverse, low-cost and portable method for controlling IE intensity aimed at reducing BP.

\section{Disclosure}

The authors report no conflicts of interest in this work.

\section{References}

1. World Health Organization. World Health Statistics: a snapshot of global health. WHO press 2012; 27(2):1-180 doi:10.2307/3348165

2. Chan M. A Global Brief on Hypertension - Silent Killer, Global Public Health Crisis. World health organ. 2013; 1(1):1-40. doi:10.1136/ bmj.1.4815.882-a

3. NHS Digital. Cardiovascular Disease. NHS website.https://www.nhs.uk/conditions/cardiovascular-disease/. Published 2015. Accessed February 11, 2016.

4. NHS Digital. Coronary Heart Disease. NHS website.https://www.nhs.uk/ conditions/coronary-heart-disease/. Published 2015. Accessed February 11, 2016.

5. Leary SD, Ness AR, Smith GD, et al. Physical activity and blood pressure in childhood: findings from a population-based study. Hypertension. 2008;51(1):92-98. doi:10.1161/HYPERTENSIONAHA.107.099051

6. Rosendorff C, Lackland DT, Allison M, et al. Treatment of Hypertension in Patients with Coronary Artery Disease: A Scientific Statement from the American Heart Association, American College of Cardiology, and American Society of Hypertension. J Hypertens. 2015; 65(6):1372-1407. doi:10.1161/HYP.0000000000000018

7. Turnbull F. Effects of different blood- pressure- lowering regimens on major cardiovascular events: results of prospectively-designed overviews of randomised trials. Lancet. 2003;362(9395):1527-1535. doi:10.1017/CBO9781107415324.004 
8. Brown MJ. Science, medicine, and the future. Hypertension. $\mathrm{Br}$ Med J. 1997;314(7089):1258-1261. doi:10.1017/CBO9781107415324.004

9. Hajjar I, Kotchen TA. Trends in prevalence, awareness, treatment, and control of hypertension in the United States, 1988-2000. J Chem Inf Model. 2003;290(2):199-206. doi:10.1017/CBO9781107415324.004

10. Rajpura J, Nayak R. Medication adherence in a sample of elderly suffering from hypertension: evaluating the influence of illness perceptions, treatment beliefs, and illness burden. J Manag Care Pharm. 2014;20(1):58-65. doi:10.18553/jmcp.2014.20.1.58

11. National institute for health and clinical excellence guidelines for the clinical management of primary hypertension in adults (update). London Natl Inst Clin Excell. 2011;127:1-94. PMID:22855971.

12. Williams B, Poulter NR, Brown MJ, et al. Guidelines for management of hypertension: report of the fourth working party of the British Hypertension Society, 2004 - BHS IV. J Hum Hypertens. 2004;18:139-185. doi:10.1038/sj.jhh.1001683

13. Pescatello LS, Franklin BA, Fagard R, Farquhar WB, Kelley GA, Ray CA. American College of Sports Medicine position stand Exercise and hypertension. Med Sci Sports Exerc. 2004;36 (3):533-553. doi:10.1017/CBO9781107415324.004

14. Whelton PK, Carey RM, Aronow WS, et al. ACC/AHA/AAPA/ABC/ $\mathrm{ACPM} / \mathrm{AGS} / \mathrm{APhA} / \mathrm{ASH} / \mathrm{ASPC} / \mathrm{NMA} / \mathrm{PCNA}$ guideline for the prevention, detection, evaluation, and management of high blood pressure in adults. J Am Coll Cardiol. 2017;2017:1-193. doi:10.1016/j. jacc.2017.11.006

15. Leung AA, Nerenberg K, Daskalopoulou SS, et al. Hypertension Canada's 2016 Canadian Hypertension Education Program Guidelines for Blood pressure measurement, diagnosis, assessment of risk, prevention, and treatment of hypertension. Can J Cardiol. 2016;32(5):569-588. doi:10.1016/j.cjca.2016.02.066

16. Mancia G, Fagard R, Narkiewicz K, et al. ESH/ESC guidelines for the management of arterial hypertension: the Task Force for the management of arterial hypertension of the European Society of Hypertension (ESH) and of the European Society of Cardiology (ESC). Eur Heart J. 2013;34(28):2159-2219. doi:10.1093/eurheartj/eht151

17. Weber MA, Schiffrin EL, White WB, et al. Clinical practice guidelines for the management of hypertension in the community a statement by the American Society of Hypertension and the International Society of Hypertension. J Clin Hypertens. 2014;16 (1):14-26. doi:10.1111/jch.12237

18. Pescatello LS, MacDonald HV, Lamberti L, Johnson BT. Exercise for hypertension: a prescription update integrating existing recommendations with emerging research. Curr Hypertens Rep. 2015;17 (87):1-10. doi:10.1007/s11906-015-0600-y

19. Inder JD, Carlson DJ, Dieberg G, Mcfarlane JR, Hess NCL, Smart NA. Isometric exercise training for blood pressure management: a systematic review and meta-analysis to optimize benefit. Hypertens Res. 2016;39(2):89-94. doi:10.1038/ hr.2015.111

20. Carlson DJ, Inder J, Palanisamy SKA, McFarlane JR, Dieberg G, Smart NA. The efficacy of isometric resistance training utilizing handgrip exercise for blood pressure management: a randomized trial. Medicine (Baltimore). 2016;95(52):e5791. doi:10.1097/ MD.0000000000005791

21. Baross AW, Hodgson DA, Padfield SL, Swaine IL. Reductions in resting blood pressure in young adults when isometric exercise is performed whilst walking. $J$ Sports Med. 2017;2017:1-6. doi: $10.1155 / 2017 / 7123834$

22. Garg R, Malhotra V, Kumar A, Dhar U, Tripathi Y. Effect of isometric handgrip exercise training on resting blood pressure in normal healthy adults. J Clin Diagnostic Res. 2014;8(9):BC08-BC10. doi:10.7860/JCDR/2014/8908.4850

23. Taylor AC, McCartney N, Kamath MV, Wiley RL. Isometric training lowers resting blood pressure and modulates autonomic control. Med Sci Sports Exerc. 2003;35(2):251-256. doi:10.1249/ 01.MSS.0000048725.15026.B5
24. Wiley RL, Dunn CL, Cox RH, Hueppchen NA, Scott MS. Isometric exercise training lowers resting blood pressure. Med Sci Sports Exerc. 1992;24(7):749-754.

25. Stiller-Moldovan C, Kenno K, McGowan CL. Effects of isometric handgrip training on blood pressure (resting and $24 \mathrm{~h}$ ambulatory) and heart rate variability in medicated hypertensive patients. Blood Press Monit. 2012;17(2):55-61. doi:10.1097/MBP.0b013e32835136fa

26. Ray CA, Carrasco DI. Isometric handgrip training reduces arterial pressure at rest without changes in sympathetic nerve activity. $\mathrm{Am}$ J Physiol Hear Circ Physiol. 2000;279:245-249. doi:10.1152/ ajpheart.2000.279.1.H245

27. Badrov MB, Bartol CL, Dibartolomeo MA, Millar PJ, McNevin NH, McGowan CL. Effects of isometric handgrip training dose on resting blood pressure and resistance vessel endothelial function in normotensive women. Eur J Appl Physiol. 2013;113(8):2091-2100. doi:10.1007/s00421-013-2644-5

28. McGowan CL, Proctor DN, Swaine IL, Brook RD, Jackson EA, Levy PD. Isometric handgrip as an adjunct for blood pressure control: a primer for clinicians. Curr Hypertens Rep. 2017;19(51):1-8. doi:10.1007/s11906-017-0748-8

29. Devereux GR, Wiles JD, Swaine IL. Reductions in resting blood pressure after 4 weeks of isometric exercise training. Eur J Appl Physiol. 2010;109(4):601-606. doi:10.1007/s00421-010-1394-x

30. Wiles JD, Goldring N, Coleman D. Home-based isometric exercise training induced reductions resting blood pressure. Eur J Appl Physiol. 2017;117(1):83-93. doi:10.1007/s00421-016-3501-0

31. Devereux GR, Wiles JD, Howden R. Immediate post-isometric exercise cardiovascular responses are associated with training-induced resting systolic blood pressure reductions. Eur J Appl Physiol. 2015;115(2):327-333. doi:10.1007/s00421-014-3021-8

32. Taylor K, Wiles J, Coleman D, Leeson P, Sharma R, O’Driscoll J. Neurohumoral and ambulatory haemodynamic adaptations following isometric exercise training in unmedicated hypertensive patients. $J$ Hypertens. 2018; Epub ahead of print. doi:10.1097/ HJH.0000000000001922.Epub

33. Gill KF, Arthur ST, Swaine I, et al. Intensity-dependent reductions in resting blood pressure following short-term isometric exercise training. J Sports Sci. 2015;33(6):616-621. doi:10.1080/02640414.2014.953979

34. Wiles JD, Coleman DA, Swaine IL. The effects of performing isometric training at two exercise intensities in healthy young males. Eur J Appl Physiol. 2010;108(3):419-428. doi:10.1007/s00421-009-1025-6

35. Baross AW, Wiles JD, Swaine IL. Double-leg isometric exercise training in older men. Open Access J Sport Med. 2013;4:33-40. doi:10.2147/OAJSM.S39375

36. Bentley DC, Thomas S. Maximal Intermittent Handgrip Strategy : Design and Evaluation of an Exercise Protocol and a Grip Tool. Clin Interv Aging. 2016;11(1):589-601.

37. Carlson DJ, Dieberg G, Hess NC, Millar PJ, Smart NA. Isometric exercise training for blood pressure management: a systematic review and meta-analysis. Mayo Clin Proc. 2014;89(3):327-334. doi:10.1016/j.mayocp.2013.10.030

38. Owen A, Wiles J, Swaine I. Effect of isometric exercise on resting blood pressure: a meta analysis. J Hum Hypertens. 2010;24 (12):796-800. doi:10.1038/jhh.2010.13

39. Somani Y, Baross A, Levy P, et al. Reductions in ambulatory blood pressure in young normotensive men and women after isometric resistance training and its relationship with cardiovascular reactivity. Blood Press Monit. 2017;22(1):1-7. doi:10.1097/MBP.0000000000000222

40. Millar PJ, Bray SR, McGowan CL, MacDonald MJ, McCartney N. Effects of isometric handgrip training among people medicated for hypertension: a multilevel analysis. Blood Press Monit. 2007;12 (5):307-314. doi:10.1097/MBP.0b013e3282cb05db

41. Millar PJ, Bray SR, MacDonald MJ, McCartney N. The hypotensive effects of isometric handgrip training using an inexpensive spring handgrip training device. J Cardiopulm Rehabil Prev. 2008;28 (3):203-207. doi:10.1097/01.HCR.0000320073.66223.a7 
42. Millar P, Paashuis A, McCartney N. Isometric handgrip effects on hypertension. Curr Hypertens Rev. 2009;5(1):54-60. doi:10.2174/ 157340209787314351

43. Bentley DC, Nguyen CH, Thomas SG. Resting blood pressure reductions following handgrip exercise training and the impact of age and sex: a systematic review and narrative synthesis. Syst Rev. 2018;7(29):1-17.

44. Baross AW, Wiles JD, Swaine IL. Effects of the intensity of leg isometric training on the vasculature of trained and untrained limbs and resting blood pressure in middle-aged men. Int J Vasc Med. 2012;1-8. doi:10.1155/2012/964697

45. Cornelissen VA, Smart NA. Exercise training for blood pressure: a systematic review and meta-analysis. $J$ Am Heart Assoc. 2013;1-68. doi:10.1161/JAHA.112.004473

46. Perri MG, Anton SD, Durning PE, et al. Adherence to exercise prescriptions : effects of prescribing moderate versus higher levels of intensity and frequency. Heal Psychol. 2002;21(5):452-458. doi:10.1037//0278-6133.21.5.452

47. King AC, Castro C, Wilcox S, Eyler AA, Sallis JF, Brownson RC. Personal and environmental factors associated with physical inactivity among different racial-ethnic groups of U.S. middle-aged and older-aged women. Heal Psychol. 2000;19(4):354-364. doi:10.1037/AB78

48. Barbour KA, Miller NH. Adherence to exercise training in heart failure : a review. Hear Fail Rev. 2008;13:81-89. doi:10.1007/s10741-007-9054-x

49. Van Assche T, Buys R, De Jaeger M, Coeckelberghs E, Cornelissen V. One single bout of low intensity isometric handgrip exercise reduces blood pressure during daily activities in healthy preand hypertensive individuals. J Sports Med Phys Fitness. 2017;57 (4):469-475. doi:10.23736/S0022-4707.16.06239-3

50. Vischer AS, Burkard T. Principles of blood pressure measurement current techniques, office vs ambulatory blood pressure measurement. Adv Exp Med Biol. 2016. doi:10.1007/5584

51. Pickering TG, Hall JE, Appel LJ, et al. Recommendations for blood pressure measurement in humans and experimental animals: part 1: blood pressure measurement in humans - A statement for professionals from the subcommittee of professional and public education of the American Heart Association Co. Circulation. 2005;111 (5):697-716. doi:10.1161/01.CIR.0000154900.76284.F6

52. Roberts HC, Dnison HJ, Martin HJ, et al. A review of the measurement of grip strength in clinical and epidemiological studies: towards a standardised approach. Age Ageing. 2011;(40):423-429. doi:10.1093/ ageing/afr051

53. Hopkins WG. Individual responses made easy. J Appl Physiol. 2015; (118):1444-1446. doi:10.1152/japplphysiol.00098.2015

54. Ray CA, Carrasco DI. Isometric handgrip training reduces arterial pressure at rest without changes in sympathetic nerve activity. Am J Physiol Heart Circ Physiol. 2000;279(1):H245-H249. doi:10.1152/ ajpheart.2000.279.1.H245

55. Peters PG, Alessio HM, Hagerman AE, Ashton T, Nagy S, Wiley RL. Short-term isometric exercise reduces systolic blood pressure in hypertensive adults: possible role of reactive oxygen species. Int J Cardiol. 2006;110(2):199-205. doi:10.1016/j.ijcard.2005.07.035

56. Hess NCL, Carlson DJ, Inder JD, Jesulola E, Mcfarlane JR, Smart NA. Clinically meaningful blood pressure reductions with low intensity isometric handgrip exercise. A randomized trial. Physiol Res. 2016;65 (3):461-468. doi:10.1017/CBO9781107415324.004
57. Hanik SE, Badrov MB, Stiller-Moldovan C, et al. 084 isometric handgrip training induces equal blood pressure reductions in normotensive males and females without influencing heart rate variability. Can J Cardiol. 2012;28(5):S118-S119. doi:10.1016/j.cjca.2012.07.094

58. Bentley DC, Nguyen CH, Thomas SG. Resting blood pressure reductions following isometric handgrip exercise training and the impact of age and sex: protocol for a systematic review. Syst Rev. 2015;4 (1):2-7. doi:10.1186/s13643-015-0164-6

59. Badrov MB, Horton S, Millar PJ, Mcgowan CL. Cardiovascular stress reactivity tasks successfully predict the hypotensive response of isometric handgrip training in hypertensives. Psychophysiology. 2013;50(4):407-414. doi:10.1111/psyp.12031

60. Page P. Clinical commentary beyond statistical significance: clinical interpretation of rehabilitation research corresponding author. Int J Sports Phys Ther. 2014;9(5):726-736.

61. Brignardello-Petersen R, Carrasco-Labra A, Shah P, Azarpazhooh A. A practitioner's guide to developing critical appraisal skills: what is the difference between clinical and statistical significance? J Am Dent Assoc. 2014;144(7):780-786. doi:10.14219/jada.archive.2013.0187

62. Copay AG, Subach BR, Glassman SD, Polly DW, Schuler TC. Understanding the minimum clinically important difference: a review of concepts and methods. Spine J. 2007;7:541-546. doi:10.1016/j.spinee.2007.01.008

63. Stamler J, Rose G, Stamler R, Elliott P, Dyer A, Marmot M. Special feature INTERSALT study findings. Hypertension. 1989;14 (5):570-577. doi:10.1161/01.HYP.14.5.570

64. Cook NR, Cohen J, Patricia HR, Taylor JO, Hennekens CH. Implications of small reductions in diastolic blood pressure for primary prevention. Arch Intern Med. 1995;155:701-709.

65. Stamler J. The INTERSALT study: background, methods, findings, and implications. Am J Clin Nutr. 1997;65:626-642. doi:10.1093/ ajen/65.2.626S

66. Badrov M, Bartol C, Millar PJ, McNevin NH. Effects of isometric handgrip training dose on resting blood pressure and resistance vessel endothelial function in normotensive women. Eur J Appl Physiol. 2013;113:2091-2100. doi:10.1007/s00421-013-2644-5

67. Devereux GR, Wiles JD, Swaine I. Markers of isometric training intensity and reductions in resting blood pressure. J Sports Sci. 2011;29(7):715-724. doi:10.1080/02640414.2011.552113

68. Howden R, Lightfoot JT, Brown SJ, Swaine IL. The effects of isometric exercise training on resting blood pressure and orthostatic tolerance in humans. Exp Physiol. 2002;87(4):507-515. doi:10.1111/ j.1469-445X.2002.tb00064.X

69. Bentley DC, Nguyen CHP, Thomas SG. High-intensity handgrip training lowers blood pressure and increases heart rate complexity among postmenopausal women : a pilot study. Blood press Monit. 2018;23(2):71-78. DOI:10.1097/MBP.0000000000000313

70. Zona health. Zona health Inc. Zona Health Inc website.

71. Dunne FP, Barry DG, Ferriss JB, Grealy G, Murphy D. Changes in blood pressure during the normal menstrual cycle. ClinicalScience. 1991;81:515-518. doi:10.1089/10799900260100150

72. Greenberg G, Imeson JD, Thompson SG, Meade TW. Blood pressure and the menstrual cycle. Br J Obstet Gynaecol. 1985;92 (10):1010-1014. doi:10.1111/j.1471-0528.1985.tb02995.x
Open Access Journal of Sports Medicine

\section{Publish your work in this journal}

Open Access Journal of Sports Medicine is an international, peerreviewed, open access journal publishing original research, reports, reviews and commentaries on all areas of sports medicine. The manuscript management system is completely online and includes a very quick and fair peer-review system. Visit http://www.dovepress. com/testimonials.php to read real quotes from published authors. 